\title{
METRIC SUBREGULARITY FOR NONCLOSED CONVEX MULTIFUNCTIONS IN NORMED SPACES*
}

\author{
XI Yin Zheng ${ }^{1}$ AND Kung Fu NG ${ }^{2}$
}

\begin{abstract}
In terms of the normal cone and the coderivative, we provide some necessary and/or sufficient conditions of metric subregularity for (not necessarily closed) convex multifunctions in normed spaces. As applications, we present some error bound results for (not necessarily lower semicontinuous) convex functions on normed spaces. These results improve and extend some existing error bound results.
\end{abstract}

Mathematics Subject Classification. 90C31, 90C25, 49J52.

Received September 13, 2008. Revised February 4, 2009.

Published online June 18, 2009.

\section{INTRODUCTION}

In this paper we discuss metric subregularity of the generalized equation

$$
b \in F(x),
$$

where $F: X \rightarrow 2^{Y}$ is a convex multifunction, $b \in Y$ is a given point, and $X, Y$ are normed spaces. Following Dontchev and Rockafellar [5], we say that (GE) is metrically subregular at $a \in F^{-1}(b)$ if there exists $\tau \in(0,+\infty)$ such that

$$
d\left(x, F^{-1}(b)\right) \leq \tau d(b, F(x)) \text { for all } x \text { close to } a .
$$

This property provides an estimate of how far a candidate $x$ can be from the solution set of generalized equation (GE). The metric subregularity has been already studied by many authors under various names (cf. $[1,7-9,13,16,22,24])$. A classical and stronger notion is metric regularity: $F$ is metrically regular at $a$ for $b$ if there exists $\tau \in(0,+\infty)$ such that

$$
d\left(x, F^{-1}(y)\right) \leq \tau d(y, F(x)) \text { for all }(x, y) \text { close to }(a, b) .
$$

The metric regularity has been well studied (for the details see $[6,12,17,19]$ and references therein).

Keywords and phrases. Metric subregularity, multifunction, normal cone, coderivative.

* This research was supported by an Earmarked Grant (GRF) from the Research Grant Council of Hong Kong and the National Natural Science Foundation of P.R. China (Grant No. 10761012).

1 Department of Mathematics, Yunnan University, Kunming 650091, P. R. China. xyzheng@ynu.edu.cn

2 Department of Mathematics, The Chinese University of Hong Kong, Shatin, New Territory, Hong Kong. kfng@math.cuhk.edu.hk 
Let $f: X \rightarrow R \cup\{+\infty\}$ be a proper convex function. Let $b=0$ and $F(x):=[f(x),+\infty)$ for all $x \in \operatorname{dom}(f)$ and $F(x)=\emptyset$ for all $x \in X \backslash \operatorname{dom}(f)$. In this special case, (GE) reduces to the following convex inequality

$$
f(x) \leq 0 .
$$

and (1.1) reduces to

$$
d(x, S) \leq \tau[f(x)]_{+} \text {for all } x \text { close to } a,
$$

where $S$ denotes the solution set of (CIE) and $[f(x)]_{+}:=\max \{f(x), 0\}$. Usually, (CIE) is said to have a local error bound at $a$ if there exists $\tau \in(0,+\infty)$ such that (1.2) holds. Error bounds have important applications in mathematical programming and have been extensively studied (cf. $[2,3,10,11,14,15,18,20])$. In particular, in terms of the normal cone and the subdifferential, many authors established necessary and/or sufficient conditions for error bounds. For example, Lewis and Pang [14] proved that if $X=R^{n}, f$ is lower semicontinuous and (CIE) has a local error bound at $a \in S$ then $N(S, a)=\operatorname{cl}\left(R_{+} \partial f(a)\right)$ (see the next section for definitions and notations). Li [15] further proved that if $X=R^{n}$ and $f(x)=\max _{1 \leq i \leq m} f_{i}(x)$ with each $f_{i}$ being convex and smooth then (CIE) has a local error bound at $a \in S$ if and only if there exists $\delta \in(0,+\infty)$ such that $N(S, x)=R_{+} \partial f(x)$ for all $x \in S \cap B(a, \delta)$, where $B(a, \delta)$ denotes the open ball with center $a$ and radius $\delta$. With the help of the approximate projection theorem for a closed convex set, the authors [23] proved that if $X$ is a Banach space and $f$ is lower semicontinuous then (CIE) has a local error bound at $a \in S$ if and only if there exist $\tau, \delta \in(0,+\infty)$ such that $N(S, x) \cap B_{X^{*}} \subset[0, \tau] \partial f(x)$ for all $x \in S \cap B(a, \delta)$, where $0 \partial f(x)$ is understood as $\partial^{\infty} f(x)$. In the case when $X$ is a Hilbert space, Burke and Deng [2] proved the same result. Zheng and Ng [24] further extended the above results to metric regularity of (GE) and proved the following result (which is useful for the later analysis).

Theorem 1.1. Let $X, Y$ be Banach spaces and $F$ be a closed convex multifunction from $X$ to $Y$. Let $b \in Y$, $a \in F^{-1}(b)$ and $\tau \in(0,+\infty)$. Then, the following statements are equivalent.

(i) There exists $\delta_{1}>0$ such that

$$
d\left(x, F^{-1}(b)\right) \leq \tau d(b, F(x)) \quad \forall x \in B\left(a, \delta_{1}\right) .
$$

(ii) There exists $\delta_{2}>0$ such that

$$
N\left(F^{-1}(b), u\right) \cap B_{X^{*}} \subset \tau D^{*} F(u, b)\left(B_{Y^{*}}\right) \quad \forall u \in F^{-1}(b) \cap B\left(a, \delta_{2}\right) .
$$

The study of the solution set of (CIE) associated to a non-lower-semicontinuous function $f$ arose from an extensive class of outer approximation methods for convex optimization (see [4] and references therein). Recently, $\mathrm{Hu}$ [11] considered error bounds for (CIE) in the case when $f$ is not necessarily lower semicontinuous. As the main results (see [11], Thms. 3.1 and 3.2), Hu [11] proved the following theorem.

Theorem 1.2. Let $X$ be a Banach space and $S$ be closed. Then, the following two assertions hold.

(i) (CIE) has a local error bound at $a \in S$ with coefficient $\tau>0$ if and only if there exists $\delta>0$ such that

$$
N(S, u) \subset[0,+\infty) \partial f(u)+\partial^{\infty} f(u) \text { and } d(0, E[\partial f(u)] \cap N(S, u)) \geq \frac{1}{\tau}
$$

for all $u \in \operatorname{bd}(A) \cap B(a, \delta)$ (the definition of $E[\partial f(u)]$ is given after Cor. 3.6).

(ii) (CIE) has a global error bound with coefficient $\tau$ if and only if (1.3) holds for all $u \in \operatorname{bd}(S)$.

In this paper, in terms of normal cone, we establish approximate projection results for a complete convex set and a convex cone in a normed space. Using these results, we provide some necessary and/or sufficient conditions (in terms of the normal cone and the coderivative) for (GE) to be metrically subregular at a point in the solution set. Our assumptions are minimal: $X, Y$ are normed spaces and $F: X \rightarrow 2^{Y}$ is (not necessarily 
closed) convex multifunction. As applications, we provide some error bound results for differentiable convex inclusions in normed spaces, which extend recent results by Burke and Deng [3]. We also give some error bound results of (CIE), applicable to proper convex (not necessarily lower semicontinuous) functions on $X$; thereby we improve and extend the above Theorem 1.2 of $\mathrm{Hu}[11]$.

\section{Preliminaries}

Throughout we assume that $X$ and $Y$ are normed spaces. Let $B_{X}$ denote the closed unit ball of $X$. For any set $A$, we use $\operatorname{cl}(A), \operatorname{int}(A)$ and $\operatorname{bd}(A)$ respectively denote the closure, interior and boundary of $A$.

For a (not necessarily closed) convex subset $A$ of $X$ and $a \in A$, we use $T(A, a)$ to denote the tangent cone of $A$ at $a$ in Bouligand's sense. Thus $v \in T(A, a)$ if and only if there exist a sequence $\left\{a_{n}\right\}$ in $A$ and a sequence $\left\{t_{n}\right\}$ of positive numbers convergent to 0 such that $\frac{a_{n}-a}{t_{n}}$ converges to $v$.

We denote by $N(A, a)$ the normal cone of $A$ at $a$, that is,

$$
N(A, a):=\left\{x^{*} \in X^{*}:\left\langle x^{*}, x-a\right\rangle \leq 0 \text { for all } x \in A\right\} .
$$

Clearly, $N(A, a)=N(T(A, a), 0)$. It is easy to verify that $T(A, a)=T(\operatorname{cl}(A), a)$ and $N(A, a)=N(\operatorname{cl}(A), a)$.

Let $F: X \rightarrow 2^{Y}$ be a multifunction; let $\operatorname{dom}(F)$ and $\operatorname{gph}(F)$ respectively denote the domain and graph of $F$, that is,

$$
\operatorname{dom}(F):=\{x \in X: F(x) \neq \emptyset\} \text { and } \operatorname{gph}(F):=\{(x, y) \in X \times Y: y \in F(x)\} .
$$

We say that $F$ is convex (resp. closed) if $\operatorname{gph}(F)$ is a convex (resp. closed) subset of $X \times Y$. It is known that $F$ is convex if and only if

$$
t F\left(x_{1}\right)+(1-t) F\left(x_{2}\right) \subset F\left(t x_{1}+(1-t) x_{2}\right) \quad \forall x_{1}, x_{2} \in X \text { and } \forall t \in[0,1] .
$$

For a (not necessarily closed) convex multifunction $F$ and $(x, y) \in \operatorname{gph}(F)$, the tangent derivative $D F(x, y)$ of $F$ at $(x, y)$ is defined by

$$
D F(x, y)(u)=\{v \in Y:(u, v) \in T(\operatorname{gph}(F),(x, y))\} \quad \forall u \in X .
$$

Let $D^{*} F(x, y)$ denote the coderivative of $F$ at $(x, y)$, which is defined by

$$
D^{*} F(x, y)\left(y^{*}\right)=\left\{x^{*} \in X^{*}:\left(x^{*},-y^{*}\right) \in N(\operatorname{gph}(F),(x, y))\right\} \quad \forall y^{*} \in Y^{*} .
$$

We will need the following results on the coderivative and normal cone.

Lemma 2.1. Let $F$ be a convex multifunction between normed spaces $X$ and $Y$. Let $b \in Y$ and $u \in F^{-1}(b)$. Then, the following statements hold.

(i) $D^{*} F(u, b)\left(Y^{*}\right) \subset N\left(F^{-1}(b), u\right)$ and $\operatorname{dom}\left(D^{*} F(u, b)\right) \subset-N(F(u), b)$.

(ii) If, in addition, $b \in \operatorname{int}(F(u))$, then

$$
N\left(F^{-1}(b), u\right)=N(\operatorname{dom}(F), u)=D^{*} F(u, b)\left(Y^{*}\right)=D^{*} F(u, b)(0) .
$$

Proof. Let $y^{*} \in Y^{*}$ and $x^{*} \in D^{*} F(u, b)\left(y^{*}\right)$. Then, by (2.1) and (2.3), one has

$$
\left\langle x^{*}, x-u\right\rangle-\left\langle y^{*}, y-b\right\rangle \leq 0 \quad \forall(x, y) \in \operatorname{gph}(F) .
$$

Noting that $F^{-1}(b) \times\{b\} \subset \operatorname{gph}(F)$ and $\{u\} \times F(u) \subset \operatorname{gph}(F)$, it follows that

$$
x^{*} \in N\left(F^{-1}(b), u\right) \text { and } y^{*} \in-N(F(u), b) .
$$


This shows that (i) holds. To prove (ii), assume that there exists $r>0$ such that $B(b, r) \subset F(u)$, and hence $N(F(u), b) \subset N(B(b, r), b)=\{0\}$. This and the second inclusion of (i) imply that $D^{*} F(u, b)\left(Y^{*}\right)=$ $D^{*} F(u, b)(0)$. Noting also that $D^{*} F(u, b)(0)=N(\operatorname{dom}(F), u) \subset N\left(F^{-1}(b), u\right)$, to prove (2.4) we need only show that

$$
N\left(F^{-1}(b), u\right) \subset N(\operatorname{dom}(F), u) .
$$

Let $u^{*} \in N\left(F^{-1}(b), u\right)$. Let $x \in \operatorname{dom}(F)$ and take $y \in F(x)$. Then, there exists $t>0$ such that $b+t(b-y) \in$ $B(b, r) \subset F(u)$. Writing $b=\frac{t}{1+t} y+\frac{1}{1+t}(b+t(b-y))$, it follows from the convexity of $F$ that $b \in F\left(\frac{t}{1+t} x+\frac{1}{1+t} u\right)$, so $\frac{t}{1+t} x+\frac{1}{1+t} u \in F^{-1}(b)$. Therefore, $\left\langle x^{*}, \frac{t}{1+t} x+\frac{1}{1+t} u\right\rangle \leq\left\langle x^{*}, u\right\rangle$ and so $\left\langle x^{*}, x\right\rangle \leq\left\langle x^{*}, u\right\rangle$. This shows that $x^{*} \in N(\operatorname{dom}(F), u)$, and $(2.5)$ is proved. The proof is completed.

Let $f: X \rightarrow R \cup\{+\infty\}$ be a proper convex function. For $x \in \operatorname{dom}(f):=\{x \in X: f(x)<+\infty\}$, let $\partial f(x)$ denote the subdifferential of $f$ at $x$, that is,

$$
\partial f(x):=\left\{x^{*} \in X^{*}:\left\langle x^{*}, z-x\right\rangle \leq f(z)-f(x) \quad \forall z \in X\right\} .
$$

It is well known and easy to verify that

$$
\partial f(x)=\left\{x^{*} \in X^{*}:\left(x^{*},-1\right) \in N(\operatorname{epi}(f),(x, f(x)))\right\},
$$

where epi $(f):=\{(x, t) \in X \times R: f(x) \leq t\}$ is the epigraph of $f$. The singular subdifferential of $f$ at $x$ is defined as

$$
\partial^{\infty} f(x):=\left\{x^{*} \in X^{*}:\left(x^{*}, 0\right) \in N(\operatorname{epi}(f),(x, f(x)))\right\} .
$$

Let $\delta_{A}$ denote the indicator function of a set $A$, that is, $\delta_{A}(x)=0$ for $x \in A$ and $\delta_{A}(x)=+\infty$ otherwise. Then $\partial \delta_{A}(x)=N(A, x)$ for all $x \in A$.

The following known result ( $c f$. [21], Thm. 2.8.3) is useful for us.

Proposition 2.2. Let $f_{1}, f_{2}: X \rightarrow R \cup\{+\infty\}$ be proper convex functions and $x_{0} \in \operatorname{dom}\left(f_{1}\right) \cap \operatorname{dom}\left(f_{2}\right)$. Suppose that either $f_{1}$ or $f_{2}$ is continuous at $x_{0}$. Then

$$
\partial\left(f_{1}+f_{2}\right)\left(x_{0}\right)=\partial f_{1}\left(x_{0}\right)+\partial f_{2}\left(x_{0}\right) .
$$

We will need the following lemmas, which supplement the approximate projection theorem on a Banach space (see [25], Thm. 3.1).

Lemma 2.3. Let $A$ be a nonempty, complete, and convex subset of $X$. Then, for any $\beta \in(0,1)$ and any $x \in X \backslash A$ there exist $z \in \operatorname{bd}(A)$ and $z^{*} \in N(A, z)$ with $\left\|z^{*}\right\|=1$ such that

$$
\beta\|x-z\|<d(x, A) \text { and } \beta\|x-z\|<\left\langle z^{*}, x-z\right\rangle .
$$

Proof. Let $x \in X \backslash A$ and define $\phi(u):=\|u-x\|$ for all $u \in A$. Take a sequence $\left\{\varepsilon_{n}\right\}$ in $(0,1)$ convergent to 0 . Since $A$ is complete, it follows from the Ekeland variational principle that there exists $z_{n} \in A$ such that

$$
\phi\left(z_{n}\right) \leq \phi(u)+\varepsilon_{n}\left\|u-z_{n}\right\| \quad \forall u \in A .
$$

This means that

$$
\left\|z_{n}-x\right\| \leq\|u-x\|+\varepsilon_{n}\left\|u-z_{n}\right\|+\delta_{A}(u) \quad \forall u \in X .
$$

Hence $0 \in \partial\left(\|\cdot-x\|+\varepsilon_{n}\left\|\cdot-z_{n}\right\|+\delta_{A}\right)\left(z_{n}\right)$. Noting that $\partial \varepsilon_{n}\left\|\cdot-z_{n}\right\|\left(z_{n}\right)=\varepsilon_{n} B_{X^{*}}$ and $\partial \delta_{A}\left(z_{n}\right)=N\left(A, z_{n}\right)$, it follows from Proposition 2.2 that $0 \in \partial\|\cdot-x\|\left(z_{n}\right)+\varepsilon_{n} B_{X^{*}}+N\left(A, z_{n}\right)$. Since $z_{n} \neq x$, it is known and easy to verify that

$$
\partial\|\cdot-x\|\left(z_{n}\right)=\left\{x^{*} \in X^{*}:\left\|x^{*}\right\|=1 \text { and }\left\langle x^{*}, z_{n}-x\right\rangle=\left\|z_{n}-x\right\|\right\} \cdot
$$


Hence there exist $x_{n}^{*}, z_{n}^{*} \in X^{*}$ such that

$$
\left\|x_{n}^{*}\right\|=1,\left\langle x_{n}^{*}, z_{n}-x\right\rangle=\left\|z_{n}-x\right\|, z_{n}^{*} \in N\left(A, z_{n}\right) \text { and }\left\|x_{n}^{*}+z_{n}^{*}\right\| \leq \varepsilon_{n} .
$$

This implies that

$$
\left(1-\varepsilon_{n}\right)\left\|z_{n}-x\right\| \leq\left\langle z_{n}^{*}, x-z_{n}\right\rangle \leq\left\|z_{n}^{*}\right\|\left\|z_{n}-x\right\|
$$

and $z_{n}^{*} \neq 0$ and so $z_{n} \in \operatorname{bd}(A)$. Let $\tilde{z}_{n}^{*}:=\frac{z_{n}^{*}}{\left\|z_{n}^{*}\right\|}$. Then

$$
\frac{\left(1-\varepsilon_{n}\right)\left\|z_{n}-x\right\|}{\left\|z_{n}^{*}\right\|} \leq\left\langle\tilde{z}_{n}^{*}, x-z_{n}\right\rangle \leq\left\|z_{n}-x\right\| .
$$

Noting that $\varepsilon_{n} \rightarrow 0,\left\|z_{n}^{*}\right\| \rightarrow 1$ and $\left\|z_{n}-x\right\| \rightarrow d(x, A)>0$ (by (2.8)), it follows from $\beta \in(0,1)$ that $\beta\left\|z_{n}-x\right\| \leq \min \left\{\left\langle\tilde{z}_{n}^{*}, x-z_{n}\right\rangle, d(x, A)\right\}$ for all $n$ large enough. The proof is completed.

Lemma 2.4. Let $C$ be a convex cone in $X$ and $x \in X$ with $d(x, C)>0$. Then there exists $x^{*} \in N(C, 0)$ such that $\left\|x^{*}\right\|=1$ and $\left\langle x^{*}, x\right\rangle=d(x, C)$.

Proof. By the separation theorem (cf. [21], Thm. 1.1.3) and the fact that $B(x, d(x, C)) \cap C=\emptyset$, there exists $x^{*} \in X^{*}$ with $\left\|x^{*}\right\|=1$ such that

$$
\inf \left\{\left\langle x^{*}, u\right\rangle: u \in B(x, d(x, C))\right\}=\sup \left\{\left\langle x^{*}, u\right\rangle: u \in C\right\}
$$

Since $C$ is a cone, it follows that

$$
\left\langle x^{*}, x\right\rangle-d(x, C)=\sup \left\{\left\langle x^{*}, u\right\rangle: u \in C\right\}=0 .
$$

This means that $x^{*} \in N(C, 0)$ and $\left\langle x^{*}, x\right\rangle=d(x, C)$. The proof is completed.

\section{The Main Results}

Let $F: X \rightarrow 2^{Y}$ be a convex multifunction and let $b$ be a given point in $Y$. Let $\tilde{X}$ and $\tilde{Y}$ denote the completions of $X$ and $Y$, respectively. Let $\tilde{F}: \tilde{X} \rightarrow 2^{\tilde{Y}}$ be defined by $\operatorname{gph}(\tilde{F})=\operatorname{cl}_{\tilde{X} \times \tilde{Y}}(\operatorname{gph}(F))$, the closure of $\operatorname{gph}(F)$ in the product space $\tilde{X} \times \tilde{Y}$. Let $u \in X$ and $r>0$. For clarity, we use notations $B_{X}(u, r):=$ $\{x \in X:\|x-u\|<r\}$ and $B_{\tilde{X}}(x, r):=\{x \in \tilde{X}:\|x-u\|<r\}$ in the following lemma.

Lemma 3.1. Let $u \in F^{-1}(b)$ and suppose that there exist $\tau, r \in(0,+\infty)$ such that

$$
d\left(x, F^{-1}(b)\right) \leq \tau d(b, F(x)) \quad \forall x \in B_{X}(u, r)
$$

Then

and

$$
\tilde{F}^{-1}(b) \cap B_{\tilde{X}}(u, r)=\mathrm{cl}_{\tilde{X}}\left(F^{-1}(b)\right) \cap B_{\tilde{X}}(u, r)
$$

$$
d\left(x, \tilde{F}^{-1}(b)\right) \leq \tau d(b, \tilde{F}(x)) \quad \forall x \in B_{\tilde{X}}\left(u, \frac{r}{2}\right) .
$$

Proof. Let $x \in B_{\tilde{X}}(u, r)$ and $y \in \tilde{F}(x)$. Then there exist a sequence $\left\{x_{n}\right\}$ in $B_{X}(u, r)$ and a sequence $\left\{y_{n}\right\}$ in $Y$ such that $x_{n} \rightarrow x, y_{n} \rightarrow y$ and $y_{n} \in F\left(x_{n}\right)$ for each $n$. It follows from (3.1) that

$$
d\left(x_{n}, F^{-1}(b)\right) \leq \tau\left\|b-y_{n}\right\| \text { for all } n .
$$

Letting $n \rightarrow \infty$, one has $d\left(x, F^{-1}(b)\right) \leq \tau\|b-y\|$. Hence,

$$
d\left(x, F^{-1}(b)\right) \leq \tau d(b, \tilde{F}(x)) \text { for any } x \in B_{\tilde{X}}(u, r) .
$$


It is now easy to verify that $\tilde{F}^{-1}(b) \cap B_{\tilde{X}}(u, r) \subset \mathrm{cl}_{\tilde{X}}\left(F^{-1}(b)\right) \cap B_{\tilde{X}}(u, r)$. Since the converse inclusion is evident (by the definition of $\tilde{F}),(3.2)$ is shown. Hence, $d\left(x, \tilde{F}^{-1}(b)\right)=d\left(x, F^{-1}(b)\right)$ for all $x \in B_{\tilde{X}}\left(u, \frac{r}{2}\right)$. This and (3.4) imply that (3.3) holds. The proof is completed.

Remark 3.1. By Lemma 3.1, the metric subregularity of $F$ implies the metric subregularity of $\tilde{F}$. But, the inverse implication is not necessarily true. Indeed, let $X=Y=\mathbb{R}$, and let $F(x)=(0,+\infty)$ for all $x \in \mathbb{R} \backslash\{0\}$ and $F(0)=[0,+\infty)$. Then, $F$ is convex and $\tilde{F}(x)=[0,+\infty)$ for all $x \in \mathbb{R}$. Note that $F^{-1}(0)=\{0\}$, $\tilde{F}^{-1}(0)=\mathbb{R}$ and $d(0, F(x))=d(0, \tilde{F}(x))=0$ for all $x \in \mathbb{R}$. Thus, $\tilde{F}$ is metrically subregular at 0 for 0 but $F$ is not metrically subregular at 0 for 0 . Hence, one can obtain necessity conditions of the metric subregularity for $F$ in terms of the results of the metric subregularity for the closed multifunction $\tilde{F}$ between two Banach spaces, but one cannot establish sufficient conditions in this line.

The following Theorems 3.2 and 3.3 extend the implications (i) $\Rightarrow$ (ii) and (ii) $\Rightarrow$ (i) in Theorem 1.1, respectively; here $X, Y$ are not necessarily Banach spaces and $F$ is not necessarily closed. For convenient, let $S_{0}:=\left\{u \in F^{-1}(b): b \notin \operatorname{int}(F(u))\right\}$.

Theorem 3.2. Let $a \in F^{-1}(b)$ and suppose that there exist $\tau, \delta \in(0,+\infty)$ such that

$$
d\left(x, F^{-1}(b)\right) \leq \tau d(b, F(x)) \quad \forall x \in B(a, \delta) .
$$

Then

equivalently

$$
N\left(F^{-1}(b), u\right) \cap B_{X^{*}} \subset \tau D^{*} F(u, b)\left(B_{Y^{*}}\right) \quad \forall u \in F^{-1}(b) \cap B(a, \delta),
$$

$$
N\left(F^{-1}(b), u\right) \cap B_{X^{*}} \subset \tau D^{*} F(u, b)\left(B_{Y^{*}}\right) \quad \forall u \in \operatorname{bd}\left(F^{-1}(b)\right) \cap S_{0} \cap B(a, \delta) .
$$

Proof. Let $u \in F^{-1}(b) \cap B(a, \delta)$ and $r:=\delta-\|u-a\|$. Then, it follows from (3.5) that (3.1) holds. Then, by Lemma 3.1, (3.2) and (3.3) hold. It follows from Theorem 1.1 that

$$
N\left(\tilde{F}^{-1}(b), u\right) \cap B_{\tilde{X}^{*}} \subset \tau D^{*} \tilde{F}(u, b)\left(B_{\tilde{Y}^{*}}\right) \text { and } N\left(\tilde{F}^{-1}(b), u\right)=N\left(\mathrm{cl}_{\tilde{X}}\left(F^{-1}(b)\right), u\right) .
$$

Noting that $X^{*}=\tilde{X}^{*}, Y^{*}=\tilde{Y}^{*}$,

$$
N(\operatorname{gph}(\tilde{F}),(u, b))=N(\operatorname{gph}(F),(u, b)) \text { and } N\left(\operatorname{cl}_{\tilde{X}}\left(F^{-1}(b)\right), u\right)=N\left(F^{-1}(b), u\right),
$$

it follows that (3.6) holds. Clearly, (3.6) implies (3.7). It remains to show that (3.7) implies (3.6). Noting that $N\left(F^{-1}(b), u\right)=\{0\}$ for any $u \in \operatorname{int}\left(F^{-1}(b)\right)$, it suffices to show that

$$
N\left(F^{-1}(b), u\right) \cap B_{X^{*}} \subset \tau D^{*} F(u, b)\left(B_{Y^{*}}\right) \quad \forall u \in \operatorname{bd}\left(F^{-1}(b)\right) \backslash S_{0} .
$$

Let $u \in \operatorname{bd}\left(F^{-1}(b)\right) \backslash S_{0}$. Then, $b \in \operatorname{int}(F(u))$. This and Lemma 2.1(ii) imply that $N\left(F^{-1}(b), u\right)=D^{*} F(u, b)(0)=$ $\tau D^{*} F(u, b)\left(B_{Y^{*}}\right)$. Hence, (3.8) holds. The proof is completed.

Next we provide sufficient conditions for $F$ to be metrically subregular at $a$ for $b$.

Theorem 3.3. Let $A$ be a complete and convex subset of $F^{-1}(b)$ and $a \in A$. Suppose that there exist $\tau, \delta \in$ $(0,+\infty)$ such that

$$
N(A, u) \cap B_{X^{*}} \subset \tau D^{*} F(u, b)\left(B_{Y^{*}}\right) \quad \forall u \in \operatorname{bd}(A) \cap B(a, \delta) .
$$

Then

$$
d(x, A) \leq \tau d(b, F(x)) \quad \forall x \in B\left(a, \frac{\delta}{2}\right),
$$

and in particular $F$ is metrically subregular at a for $b$. 
Proof. The last assertion follows trivially from (3.10). To prove (3.10), let $x \in B\left(a, \frac{\delta}{2}\right) \backslash A$. Then, $d(x, A) \leq$ $\|x-a\|<\frac{\delta}{2}$. Let $\beta \in\left(\frac{2 d(x, A)}{\delta}, 1\right)$. Then, by Lemma 2.3 there exist $u \in \operatorname{bd}(A)$ and $x^{*} \in N(A, u)$ with $\left\|x^{*}\right\|=1$ such that $\beta\|x-u\| \leq d(x, A)$ and

$$
\beta\|x-u\| \leq\left\langle x^{*}, x-u\right\rangle \cdot
$$

Thus, $\|x-u\|<\frac{\delta}{2}$ and so $\|u-a\| \leq\|u-x\|+\|x-a\|<\delta$. By (3.9) it follows that there exists $y^{*} \in B_{Y^{*}}$ such that $x^{*} \in \tau D^{*} F(u, b)\left(y^{*}\right)$. Hence,

$$
\left\langle x^{*}, x-u\right\rangle \leq \tau\left\langle y^{*}, y-b\right\rangle \leq \tau\|y-b\| \quad \forall y \in F(x) .
$$

This and (3.11) imply that $\beta\|x-u\| \leq \tau d(b, F(x))$. Since $u \in A$ we then have $\beta d(x, A) \leq \tau d(b, F(x))$. Letting $\beta \rightarrow 1^{-}$, one sees that (3.10) holds. The proof is completed.

In view of Theorem 3.3, we have the following global result.

Corollary 3.4. Let $A$ be a nonempty, complete, and convex subset of $F^{-1}(b)$ and $\tau$ be a positive constant. Suppose that

$$
N(A, u) \cap B_{X^{*}} \subset \tau D^{*} F(u, b)\left(B_{Y^{*}}\right) \quad \forall u \in \operatorname{bd}(A) .
$$

Then

$$
d(x, A) \leq \tau d(b, F(x)) \quad \forall x \in X .
$$

Proof. Let $x \in X$ and take $a \in \operatorname{bd}(A)$. Let $\delta:=2\|x-a\|+1$. Then, $x \in B\left(a, \frac{\delta}{2}\right)$ and, by the assumption, (3.9) holds. It follows from Theorem 3.3 that $d(x, A) \leq \tau d(b, F(x))$. The proof is completed.

Without the completeness of $A$, we have the following result.

Theorem 3.5. Let $A$ be a convex subset of $F^{-1}(b)$ and $a \in A$. Suppose that there exist $\delta>0$ and a convex cone $C$ in $X$ such that

$$
A \cap B(a, \delta)=(a+C) \cap B(a, \delta) .
$$

Further suppose that there exists $\tau \in(0,+\infty)$ such that

$$
N(A, a) \cap B_{X^{*}} \subset \tau D^{*} F(a, b)\left(B_{Y^{*}}\right) .
$$

Then (3.10) holds.

Proof. By (3.12), we have

$$
N(A, a)=N(a+C, a)=N(C, 0) .
$$

Let $x \in B\left(a, \frac{\delta}{2}\right)$ be such that $d(x, A)>0$. Then,

$$
d(x, A) \leq\|x-a\|<\frac{\delta}{2} \quad \text { and } \quad d(x, X \backslash B(a, \delta)) \geq \frac{\delta}{2} .
$$

Hence

$$
d(x, A)=d(x, A \cap B(a, \delta)) \text { and } d(x, a+C)=d(x,(a+C) \cap B(a, \delta)) .
$$

It follows from (3.12) that $0<d(x, A)=d(x, a+C)=d(x-a, C)$. By this and (3.14), one can apply Lemma 2.4 to find $x^{*} \in N(A, a)$ with $\left\|x^{*}\right\|=1$ such that $\left\langle x^{*}, x-a\right\rangle=d(x, A)$. By (3.13), there exists $y^{*} \in B_{Y^{*}}$ such that $x^{*} \in D^{*} F(a, b)\left(\tau y^{*}\right)$. Then, for all $y \in F(x),\left\langle x^{*}, x-a\right\rangle+\left\langle-\tau y^{*}, y-b\right\rangle \leq 0$, and hence $d(x, A) \leq \tau\|y-b\|$. Therefore $d(x, A) \leq \tau d(b, F(x))$ and (3.10) is shown. 
Corollary 3.6. Let $C$ be a convex cone in $X$ and $a \in F^{-1}(b)$. Suppose that there exist $\tau, \delta \in(0,+\infty)$ such that $(a+C) \cap B(a, \delta) \subset F^{-1}(b)$ and

$$
N(C, 0) \cap B_{X^{*}} \subset \tau D^{*} F(a, b)\left(B_{Y^{*}}\right) .
$$

Then

$$
d\left(x, F^{-1}(b)\right) \leq \tau d(b, F(x)) \quad \forall x \in B\left(a, \frac{\delta}{2}\right) .
$$

Proof. Let $A:=(a+C) \cap F^{-1}(b)$. Clearly (3.15) holds if (3.10) holds. Thus, by the second inclusion assumption and Theorem 3.5, we need only show that $N(A, a)=N(C, 0)$. But this is evident by the fact that $A \cap B(a, \delta)=(a+C) \cap B(a, \delta)$ and $N(a+C, a)=N(C, 0)$ (thanks to the first inclusion assumption). The proof is completed.

The following result shows that, for any $u \in A$, the inclusion relation given in (3.9) can be described equivalently in many useful ways. To do this, we need the following notion. For a subset $C$ of $X$, let

$$
E[C]:=\{z \in \operatorname{cl}([0,1] C): t z \notin \operatorname{cl}([0,1] C) \quad \forall t>1\}
$$

(which was introduced in [23]). In [10,11], $E[C]$ is called the end set of $C$. Hu [11] shown (cf. [11], Lem. 1.1) that if $C$ is closed and convex then

$$
E(C)=\{z \in C: t z \notin C \quad \forall t>1\} .
$$

We use $\Sigma_{Y^{*}}$ to denote the unit sphere of $Y^{*}$.

Proposition 3.7. Let $A$ be a convex subset of $F^{-1}(b)$ and $u \in A$. Let $\tau$ be a positive constant. Then the following statements are equivalent.

(i) $N(A, u) \cap B_{X^{*}} \subset \tau D^{*} F(u, b)\left(B_{Y^{*}}\right)$.

(ii) $N(A, u) \subset D^{*} F(u, b)\left(Y^{*}\right)$ and $d\left(0, E\left[D^{*} F(u, b)\left(\Sigma_{Y^{*}}\right)\right]\right) \geq \frac{1}{\tau}$.

(iii) $N(A, u)=D^{*} F(u, b)\left(Y^{*}\right)$ and $d\left(0, E\left[D^{*} F(u, b)\left(\Sigma_{Y^{*}}\right)\right]\right) \geq \frac{1}{\tau}$.

(iv) $d(h, T(A, u)) \leq \tau d(0, D F(u, b)(h))$ for all $h \in X$.

Proof. The given assumption implies that $N\left(F^{-1}(b), u\right) \subset N(A, u)$, and it follows from Lemma 2.1 that

$$
D^{*} F(u, b)\left(Y^{*}\right) \subset N(A, u) .
$$

Hence, (ii) $\Leftrightarrow$ (iii) holds.

(i) $\Rightarrow$ (ii). Clearly, (i) implies that $N(A, u) \subset D^{*} F(u, b)\left(Y^{*}\right)$. Let $z^{*}$ be an arbitrary point in $\left.E\left[D^{*} F(u, b)\left(\Sigma_{Y^{*}}\right)\right)\right]$. Then there exist $t_{n} \in(0,1], y_{n}^{*} \in \Sigma_{Y^{*}}$ and $x_{n}^{*} \in D^{*} F(u, b)\left(y_{n}^{*}\right)$ such that $t_{n} x_{n}^{*} \rightarrow z^{*}$. We claim that

$$
z^{*} \notin D^{*} F(u, b)(0) .
$$

If this is not the case, then $\frac{z^{*}}{t_{n}} \in D^{*} F(u, b)(0)$ and so $x_{n}^{*}+\frac{1}{t_{n}} z^{*} \in D^{*} F(u, b)\left(y_{n}^{*}\right)$; hence

$$
t_{n}\left(x_{n}^{*}+\frac{1}{t_{n}} z^{*}\right) \longrightarrow 2 z^{*} \in \operatorname{cl}\left([0,1] D^{*} F(u, b)\left(\Sigma_{Y^{*}}\right)\right),
$$

contradicting $z^{*} \in E\left[D^{*} F(u, b)\left(\Sigma_{Y^{*}}\right)\right]$. Since $B_{Y^{*}}$ is compact with respect to the weak ${ }^{*}$ topology $w^{*}$, without loss of generality we assume that $t_{n} y_{n}^{*} \stackrel{w^{*}}{\rightarrow} y^{*}$ for some $y^{*} \in Y^{*}$ (passing to a generalized subsequence if necessary). 
Hence, $\left(t_{n} x_{n}^{*},-t_{n} y_{n}^{*}\right) \stackrel{w^{*}}{\longrightarrow}\left(z^{*},-y^{*}\right)$. Since $\operatorname{gph}\left(D^{*} F(a, b)\right)$ is weakly* closed, it follows that $z^{*} \in D^{*} F(u, b)\left(y^{*}\right)$. This and (3.17) imply that $z^{*} \in N(A, u)$. By (i) and (3.18), one has

$$
\frac{z^{*}}{\tau\left\|z^{*}\right\|} \in D^{*} F(u, b)\left(B_{Y^{*}} \backslash\{0\}\right) \subset[0,1] D^{*} F(u, b)\left(\Sigma_{Y^{*}}\right)
$$

Hence, $\left\|z^{*}\right\| \geq \frac{1}{\tau}$ (because $z^{*} \in E\left[D^{*} F(u, b)\left(\Sigma_{Y^{*}}\right)\right]$ ). This shows that (i) $\Rightarrow$ (ii) holds.

(ii) $\Rightarrow$ (i). Let $x^{*} \in N(A, u) \cap B_{X^{*}}$. It is clear that $x^{*} \in \tau D^{*} F(u, b)\left(B_{Y^{*}}\right)$ if $x^{*} \in D^{*} F(u, b)(0)$. We can therefore assume that $x^{*} \notin D^{*} F(u, b)(0)$. Then the inclusion of (ii) implies that $x^{*} \in D^{*} F(u, b)\left(Y^{*} \backslash\{0\}\right)$. Let

$$
\lambda:=\sup \left\{t>0: t x^{*} \in \operatorname{cl}\left([0,1] D^{*} F(u, b)\left(\Sigma_{Y^{*}}\right)\right)\right\}
$$

Then, $0<\lambda<+\infty$ (because the graph of $D^{*} F(u, b)$ is closed). We claim that $\lambda \geq \frac{1}{\tau}$. Indeed, by the definition of $\lambda$, one has $\lambda x^{*} \in \operatorname{cl}\left([0,1] D^{*} F(u, b)\left(\Sigma_{Y^{*}}\right)\right)$ and $t x^{*} \notin \operatorname{cl}\left([0,1] D^{*} F(u, b)\left(\Sigma_{Y^{*}}\right)\right)$ for all $t>\lambda$. Thus, $\lambda x^{*} \in E\left[D^{*} F(u, b)\left(\Sigma_{Y^{*}}\right)\right]$. It follows from the inequality of (ii) that $\frac{1}{\tau} \leq\left\|\lambda x^{*}\right\| \leq \lambda$. This implies that

$$
\frac{1}{\tau} x^{*} \in \operatorname{cl}\left([0,1] D^{*} F(u, b)\left(\Sigma_{Y^{*}}\right)\right) \text {. }
$$

Since $N(\operatorname{gph}(F),(u, b))$ is weak ${ }^{*}$ closed and $B_{Y^{*}}$ is weak ${ }^{*}$ compact, $D^{*} F(u, b)\left(B_{Y^{*}}\right)$ is weak ${ }^{*}$ closed (by (2.3)). Hence $\operatorname{cl}\left([0,1] D^{*} F(u, b)\left(\Sigma_{Y^{*}}\right)\right) \subset D^{*} F(u, b)\left(B_{Y^{*}}\right)$. It follows from (3.19) that $x^{*} \in \tau D^{*} F(u, b)\left(B_{Y^{*}}\right)$. This shows that (ii) $\Rightarrow$ (i) holds.

(iv) $\Rightarrow(\mathrm{i})$. Note by the definitions that

$$
T(A, u) \subset T\left(F^{-1}(b), u\right) \subset(D F(u, b))^{-1}(0) .
$$

Thus, since $T(A, u)$ is closed, (iv) implies that $T(A, u)=(D F(u, b))^{-1}(0)$ and that

$$
d\left(h,(D F(u, b))^{-1}(0)\right) \leq \tau d(0, D F(u, b)(h)) \quad \forall h \in X .
$$

It follows from Theorem 3.2 (applied to $D F(u, b)$ and $(0,0)$ respectively in place of $F$ and $(a, b))$ that

$$
N\left(D F(u, b)^{-1}(0), 0\right) \cap B_{X^{*}} \subset \tau D^{*}(D F(u, b))(0,0)\left(B_{Y^{*}}\right) .
$$

This means that (i) holds because

$$
N(A, u)=N(T(A, u), 0)=N\left(D F(u, b)^{-1}(0), 0\right)
$$

and

$$
D^{*} F(u, b)=D^{*}(D F(u, b))(0,0)
$$

(i) $\Rightarrow$ (iv). By (3.21) and (3.22), (i) means that

$$
N(T(A, u), 0) \cap B_{X^{*}} \subset \tau D^{*}(D F(u, b))(0,0)\left(B_{Y^{*}}\right) .
$$

Thus, with an arbitrary $\delta>0$ and the help of (3.20), one can apply Theorem 3.5 to $T(A, u), D F(u, b)$ and $(0,0)$ in place of $C, F$ and $(a, b)$, and we conclude that

$$
d(h, T(A, u)) \leq \tau d(0, D F(u, b)(h)) \quad \forall h \in B\left(0, \frac{\delta}{2}\right) .
$$

Since $\delta>0$ is arbitrary, (iv) is seen to hold. The proof is completed. 
In view of Theorems 3.2 and 3.3 and Proposition 3.7, we have the following characterizations of the metric subregularity of $F$ at $a$ for $b$.

Corollary 3.8. Let $\tau$ be a positive constant and $a \in F^{-1}(b)$. Consider the following statements.

(i) $F$ is metrically subregular at a for $b$ with coefficient $\tau$.

(ii) There exists $\delta \in(0,+\infty)$ such that (3.6) holds.

(iii) There exists $\delta \in(0,+\infty)$ such that (3.7) holds.

(iv) There exists $\delta \in(0,+\infty)$ such that $N\left(F^{-1}(b), u\right)=D^{*} F(u, b)\left(Y^{*}\right)$ and

$$
d\left(0, E\left[D^{*} F(u, b)\left(\Sigma_{Y^{*}}\right)\right]\right) \geq \frac{1}{\tau}
$$

for any $u \in F^{-1}(b) \cap B(a, \delta)$.

(v) There exists $\delta \in(0,+\infty)$ such that $N\left(F^{-1}(b), u\right) \subset D^{*} F(u, b)\left(Y^{*}\right)$ and (3.23) hold for any $u \in$ $\operatorname{bd}\left(F^{-1}(b)\right) \cap S_{0} \cap B(a, \delta)$.

(vi) There exist $\tau, \delta \in(0,+\infty)$ such that $d\left(h, T\left(F^{-1}(b), u\right)\right) \leq \tau d(0, D F(u, b)(h))$ for any $u \in \operatorname{bd}\left(F^{-1}(b)\right) \cap$ $S_{0} \cap B(a, \delta)$ and any $h \in X$.

Then $(i) \Rightarrow(i i) \Leftrightarrow($ iii $) \Leftrightarrow(i v) \Leftrightarrow(v) \Leftrightarrow(v i)$. If $F^{-1}(b)$ is complete then (i)-(vi) are mutually equivalent.

\section{Application to ERROR BOUND FOR CONVEX INEQUALITIES}

Recall that $X$ is a normed space, $f$ is a proper (not necessarily lower semicontinuous) convex function on $X$ and that $S$ is the solution set of the corresponding convex inequality (CIE). Let

$$
F(x)=[f(x),+\infty) \quad \forall x \in X
$$

Then,

$$
S=F^{-1}(0), \quad[f(x)]_{+}=d(0, F(x)) \quad \forall x \in X,
$$

and $\operatorname{gph}(F)=\operatorname{epi}(f)$. Hence, by the definition of the coderivative, (2.6) and (2.7), it is easy to verify that for any $u \in \operatorname{dom}(f)$,

and

$$
\operatorname{dom}\left(D^{*} F(u, \alpha)\right)= \begin{cases}\mathbb{R}_{+}, & \text {if } \alpha=f(u) \\ \{0\}, & \text { if } \alpha>f(u)\end{cases}
$$

$$
D^{*} F(u, \alpha)(t)= \begin{cases}t \partial f(u), & \text { if } \alpha=f(u) \text { and } t \in \mathbb{R}_{+} \\ \partial^{\infty} f(u), & \text { if } \alpha>f(u) \text { and } t=0\end{cases}
$$

where $0 \partial f(u)$ is understood as $\partial^{\infty} f(u)$; hence

$$
D^{*} F(u, \alpha)\left(B_{\mathbb{R}}\right)= \begin{cases}{[0,1] \partial f(u),} & \text { if } \alpha=f(u) \\ \partial^{\infty} f(u), & \text { if } \alpha>f(u)\end{cases}
$$

and

$$
D^{*} F(u, \alpha)\left(\Sigma_{\mathbb{R}}\right)= \begin{cases}\partial f(u), & \text { if } \alpha=f(u) \\ \emptyset, & \text { if } \alpha>f(u) .\end{cases}
$$

By (4.2), it is clear that (CIE) has a local error bound at $a \in S$ if and only if $F$ is metrically subregular at $a$ for 0 . In view of this equivalence and the main results established in Section 3, we can provide some local error bound results for (CIE).

Proposition 4.1. Let $a \in S$ and suppose that there exist $\tau, \delta \in(0,+\infty)$ such that

$$
d(x, S) \leq \tau[f(x)]_{+} \quad \forall x \in B(a, \delta) .
$$


Then

for all $u \in S \cap B(a, \delta)$.

$$
N(S, u) \cap B_{X^{*}} \subset[0, \tau] \partial f(u)
$$

Proof. Let $F$ be as in (4.1) and $b=0$. Then, (4.5) means (3.5). It follows from Theorem 3.2 that (3.6) holds. It follows from (4.3) that (4.6) holds. This completes the proof.

Proposition 4.2. Let $A$ be a complete convex subset of $S$ with $a \in A$ and $A_{0}:=\{u \in \operatorname{bd}(A): f(u)=0\}$. Suppose that there exist $\tau, \delta \in(0,+\infty)$ such that

$$
N(A, u)=\partial^{\infty} f(u) \quad \forall u \in\left(\operatorname{bd}(A) \backslash A_{0}\right) \cap B(a, \delta)
$$

and

$$
N(A, u) \cap B_{X^{*}} \subset[0, \tau] \partial f(u) \quad \forall u \in A_{0} \cap B(a, \delta)
$$

Then

$$
d(x, A) \leq \tau[f(x)]_{+} \quad \forall x \in B\left(a, \frac{\delta}{2}\right) .
$$

Consequently, (CIE) has a local error bound at a.

Proof. Let $F$ be as in (4.1) and $b=0$. Then, by (4.3), it is clear that (4.7) and (4.8) imply (3.9). It follows from Theorem 3.3 that (3.10) holds. This means that (4.9) holds (by the second equality of (4.2)).

Lemma 4.3. Let $u \in S$. Then

$$
E[\partial f(u)] \cap N(S, u)= \begin{cases}\emptyset & f(u)<0 \\ E[\partial f(u)] & f(u)=0 .\end{cases}
$$

Proof. Without loss of generality, we assume that $\partial f(u) \neq \emptyset$. Since $\partial f(u)$ is a closed convex set, (3.16) implies that

$$
E[\partial f(u)]=\left\{x^{*} \in \partial f(u): t x^{*} \notin \partial f(u) \text { for all } t>1\right\} \text {. }
$$

In the case when $f(u)=0$, one has $\partial f(u) \subset N(S, u)$; this and (4.10) imply that $E[\partial f(u)] \cap N(S, u)=E[\partial f(u)]$. Next assume that $f(u)<0$. Then, by Lemma 2.1 (with $F$ being as in (4.1) and $b=0$ ), one has $N(S, u)=$ $\partial^{\infty} f(u)$. Since $\partial f(u)=\partial f(u)+\partial^{\infty} f(u)=\partial f(u)+\mathbb{R}_{+} \partial^{\infty} f(u)$, it follows from (4.10) that $E[\partial f(u)] \cap \partial^{\infty} f(u)=\emptyset$, and so $E[\partial f(u)] \cap N(S, u)=\emptyset$. The proof is completed.

By Lemma 4.3 and (4.4), it is clear that

$$
E\left(D^{*} F(u, 0)\left(\Sigma_{\mathbb{R}}\right)\right)=E(\partial f(u)) \cap N(S, u) \quad \forall u \in S .
$$

By this and (4.3), one can see that the following result is a special case of Corollary 3.8.

Proposition 4.4. Let $\tau$ be a positive constant. Suppose that the solution set $S$ is complete and a $\in$. Then the following statements are equivalent.

(i) (CIE) has a local error bound at a with coefficient $\tau$.

(ii) There exists $\delta \in(0,+\infty)$ such that (4.6) holds for all $u \in \operatorname{bd}(S) \cap B(a, \delta)$.

(iii) There exists $\delta \in(0,+\infty)$ such that (4.6) holds for any $u \in \operatorname{bd}(S) \cap B(a, \delta)$ with $f(u)=0$.

(iv) There exists $\delta \in(0,+\infty)$ such that

$$
N(S, u) \subset[0,+\infty) \partial f(u) \text { and } d(0, E[\partial f(u)] \cap N(S, u)) \geq \frac{1}{\tau}
$$

for any $u \in \operatorname{bd}(S) \cap B(a, \delta)$.

(v) There exists $\delta \in(0,+\infty)$ such that (4.12) holds for any $u \in \operatorname{bd}(S) \cap B(a, \delta)$ with $f(u)=0$. 
(vi) There exists $\delta \in(0,+\infty)$ such that for any $u \in \operatorname{bd}(S) \cap B(a, \delta)$ with $f(u)=0$

$$
d(h, T(S, u)) \leq \tau\left[d^{+} f(u)(h)\right]_{+} \quad \forall h \in X
$$

$$
\text { where } d^{+} f(u)(h):=\lim _{t \rightarrow 0^{+}} \frac{f(u+t h)-f(u)}{t} .
$$

Because possibly different constants $\delta$ are involved in preceding proposition, the following proposition concerning global error bounds is not a direct consequence of Proposition 4.4 but can be easily deduced by virtue of Theorem 3.2, Corollary 3.4 and Proposition 3.7.

Proposition 4.5. Let $\tau$ be a positive constant and suppose that $S$ is complete. Then, the following assertions are equivalent.

(i) $d(x, S) \leq \tau[f(x)]_{+}$for all $x \in X$.

(ii) (4.6) holds for all $u \in S$.

(iii) (4.6) holds for all $u \in \operatorname{bd}(S)$ with $f(u)=0$.

(iv) (4.12) holds for all $u \in \operatorname{bd}(S)$.

(v) (4.12) holds for all $u \in \operatorname{bd}(S)$ with $f(u)=0$.

(vi) (4.13) holds for all $u \in \operatorname{bd}(S)$ with $f(u)=0$.

Remark. Thus purely based on considering only these boundary points $u$ (of the solution set $S$ ) with $f(u)=0$ one can determine whether (CIE) has an error bound or not. Further, since

$$
[0,+\infty) \partial f(u)=[0,+\infty) \partial f(u)+\partial^{\infty} f(u) \quad \forall u \in S
$$

we see that (i) $\Leftrightarrow$ (iv) of Proposition 4.4 is an extension of (i) in Hu's Theorem 1.2 while (i) $\Leftrightarrow$ (iv) of Proposition 4.5 extends (ii) in Theorem 1.2.

\section{Application to ERROR BOUNDS FOR DifFERENTIABLE CONVEX INCLUSION}

Let $X, Y$ be normed spaces, $h: X \rightarrow Y$ be a mapping and let $C$ be a nonempty subset of $Y$. Consider the following inclusion

$$
h(x) \in C .
$$

Let $\Sigma:=\{x \in X: h(x) \in C\}$. We say that (5.1) has a local error bound at $\bar{x} \in \Sigma$ if there exist $\tau \in(0,+\infty)$ and a neighborhood $U$ of $\bar{x}$ such that

$$
d(x, \Sigma) \leq \tau d(h(x), C) \quad \forall x \in U
$$

We say that (5.1) has a global error bound if (5.2) holds for some $\tau \in(0,+\infty)$ and $U=X$.

Recall that a mapping $h: X \rightarrow Y$ is Gateaux differentiable at $\bar{x} \in X$ if there exists a bounded linear operator $h^{\prime}(\bar{x}): X \rightarrow Y$, which is called the Gateaux derivative of $h$ at $\bar{x}$, such that

$$
\lim _{t \rightarrow 0^{+}} \frac{h(\bar{x}+t d)-h(\bar{x})}{t}=h^{\prime}(\bar{x})(d) \quad \forall d \in X
$$

Recently, as one of main results (see [3], Thm. 2), Burke and Deng [3] proved that the following theorem ( $C^{\infty}$ denotes the recession cone of a closed convex set as usual). 
Theorem 5.1. Let $\bar{x} \in \Sigma$ and suppose that the following assumptions are satisfied.

(A1) $X$ is a Hilbert space.

(A2) $h$ is Gateaux differentiable at every point of $\Sigma$.

(A3) The set $C$ is a closed convex set and the function $x \mapsto d(h(x), C)$ is lower semicontinuous on $X$.

(A4) $h\left(t x_{1}+(1-t) x_{2}\right)-\left(t h\left(x_{1}\right)+(1-t) h\left(x_{2}\right)\right) \in C^{\infty} \quad \forall\left(x_{1}, x_{2}, t\right) \in X \times X \times[0,1]$.

Then, (5.1) has a local error bound at $\bar{x}$ if and only if there exist $\tau, \delta \in(0,+\infty)$ such that

$$
N(\Sigma, x)=h^{\prime}(x)^{*}(N(C, h(x)))
$$

and

$$
h^{\prime}(x)^{*}(N(C, h(x))) \cap B_{X^{*}} \subset \tau h^{\prime}(x)^{*}\left(N(C, h(x)) \cap B_{Y^{*}}\right)
$$

for all $x \in \Sigma \cap B(\bar{x}, \delta)$.

Remark. Burke and Deng [3] didn't explicitly require the lower semicontinuity assumption in (A3) but their proof is based on [2], Theorems 2.3 and 2.5 (see [3], Thm. 2 and its proof), while the later requires the semicontinuity assumption. Since (A4) implies that the function $x \mapsto d(h(x), C)$ is convex, (A3) and (A4) imply that the function is continuous.

The following Theorem 5.2 is due to $\mathrm{Li}$ [15], and can be alternatively proved via Theorem 5.1 as done by Burke and Deng [3]. The main aim of this section is to extend the above Theorem 5.1 of Burke and Deng; also our Proposition 5.6 is an improved and extended version of Theorem 5.2 (infinite dimensional setting and with weaker assumptions).

Theorem 5.2. Let $f_{1}, \ldots, f_{m}: \mathbb{R}^{n} \rightarrow \mathbb{R}$ be smooth and convex functions and $f(x)=\max \left\{f_{i}(x): i=1, \ldots, m\right\}$ for all $x \in \mathbb{R}^{n}$. Then the convex inequality (CIE) associated to $f$ has a local error bound at $\bar{x} \in S$ if and only if there exists a neighborhood $U$ of $\bar{x}$ such that (4.6) holds for all $u \in S \cap U$.

For the rest of the paper, we consider inclusion (5.1) with $X, Y, h, C$ and $\Sigma$ as at the beginning of this section. We further assume that $C$ is convex (not necessarily closed).

Lemma 5.3. Let $\bar{x}$ be a point in the solution set $\Sigma$ such that $h$ is Gateaux differentiable at $\bar{x}$. Let $F: X \rightarrow 2^{Y}$ be defined by $F(x):=h(x)-C$ for all $x \in X$ and suppose that $F$ is convex. Then

$$
\begin{gathered}
D F(\bar{x}, 0)(u)=h^{\prime}(\bar{x})(u)-T(C, h(\bar{x})) \quad \forall u \in X, \\
D^{*} F(\bar{x}, 0)\left(y^{*}\right)= \begin{cases}\left\{h^{\prime}(\bar{x})^{*}\left(y^{*}\right)\right\} & y^{*} \in N(C, h(\bar{x})) \\
\emptyset & \text { otherwise, }\end{cases}
\end{gathered}
$$

and consequently

$$
D^{*} F(\bar{x}, 0)\left(B_{Y^{*}}\right)=h^{\prime}(\bar{x})\left(N(C, h(\bar{x})) \cap B_{Y^{*}}\right)
$$

where $h^{\prime}(\bar{x})^{*}$ denotes the conjugate operator of $h^{\prime}(\bar{x})$.

Proof. Let $y^{*} \in N(C, h(\bar{x}))$. Then,

$$
\left\langle y^{*}, c-h(\bar{x})\right\rangle \leq 0 \quad \forall c \in C .
$$

We claim that

$$
h^{\prime}(\bar{x})^{*}\left(y^{*}\right) \in D^{*} F(\bar{x}, 0)\left(y^{*}\right),
$$

equivalently

$$
\left\langle h^{\prime}(\bar{x})^{*}\left(y^{*}\right), d\right\rangle-\left\langle y^{*}, h(\bar{x}+d)-c\right\rangle \leq 0 \quad \forall(d, c) \in X \times C .
$$

To do this, by (5.8), we need only show that

$$
\left\langle h^{\prime}(\bar{x})\left(y^{*}\right), d\right\rangle-\left\langle y^{*}, h(\bar{x}+d)-h(\bar{x})\right\rangle \leq 0 \quad \forall d \in X .
$$


Let $t \in(0,1)$. Then, by the convexity of $F$, one has

$$
t(h(\bar{x}+d)-C)+(1-t)(h(\bar{x})-C) \subset h(\bar{x}+t d)-C .
$$

It follows from $h(\bar{x}) \in C$ that

$$
\frac{h(\bar{x}+t d)-h(\bar{x})}{t} \in h(\bar{x}+d)-h(\bar{x})+\frac{C-h(\bar{x})}{t} .
$$

This and (5.8) imply that

$$
\left\langle y^{*}, \frac{h(\bar{x}+t d)-h(\bar{x})}{t}\right\rangle \leq\left\langle y^{*}, h(\bar{x}+d)-h(\bar{x})\right\rangle .
$$

Letting $t \rightarrow 0^{+}$, one sees that (5.10) holds. Let $v^{*} \in Y^{*}$ and $u^{*} \in D^{*} F(\bar{x}, 0)\left(v^{*}\right)$. Then, by (5.9), to prove (5.6), it suffices to show that $v^{*} \in N(C, h(\bar{x}))$ and $u^{*}=\left(h^{\prime}(\bar{x})\right)^{*}\left(v^{*}\right)$. Note that

$$
\left\langle u^{*}, d\right\rangle-\left\langle v^{*}, h(\bar{x}+d)-c\right\rangle \leq 0 \quad \forall(d, c) \in X \times C .
$$

It follows that $v^{*} \in N(C, h(\bar{x}))$ (by letting $\left.d=0\right)$ and

$$
\left\langle u^{*}, d\right\rangle-\left\langle v^{*}, h(\bar{x}+d)-h(\bar{x})\right\rangle \leq 0 \quad \forall d \in X .
$$

Hence

$$
\left\langle u^{*}, d\right\rangle \leq\left\langle v^{*}, \frac{h(\bar{x}+t d)-h(\bar{x})}{t}\right\rangle \quad \forall(d, t) \in X \times(0,+\infty) .
$$

Letting $t \rightarrow 0^{+}$, it follows that $\left\langle u^{*}, d\right\rangle \leq\left\langle v^{*}, h^{\prime}(\bar{x})(d)\right\rangle$ for all $d \in X$. This means that $u^{*}=\left(h^{\prime}(\bar{x})\right)^{*}\left(v^{*}\right)$. Thus, (5.6) is shown.

Since

$$
\operatorname{gph}(D F(\bar{x}, 0))=\left\{(x, y):\left\langle x^{*}, x\right\rangle+\left\langle y^{*}, y\right\rangle \leq 0 \forall\left(x^{*}, y^{*}\right) \in N(\operatorname{gph}(F),(\bar{x}, 0))\right\},
$$

(5.6) implies that

$$
\begin{aligned}
\operatorname{gph}(D F(\bar{x}, 0)) & =\left\{(x, y):\left\langle h^{\prime}(\bar{x})^{*}\left(y^{*}\right), x\right\rangle-\left\langle y^{*}, y\right\rangle \leq 0 \quad \forall y^{*} \in N(C, h(\bar{x}))\right\} \\
& =\left\{(x, y):\left\langle y^{*}, h^{\prime}(\bar{x})(x)-y\right\rangle \leq 0 \quad \forall y^{*} \in N(C, h(\bar{x}))\right\} \\
& =\left\{(x, y): h^{\prime}(\bar{x})(x)-y \in T(C, h(\bar{x}))\right\}
\end{aligned}
$$

This means that (5.5) holds. The proof is completed.

By virtue of (5.7), the following theorem is immediate from Corollary 3.8 (applied to $F(x)=h(x)-C$ and $b=0)$.

Theorem 5.4. Let $\bar{x} \in \Sigma$ and $h$ be Gateaux differentiable at each point of $\Sigma$. Suppose that the multifunction $x \mapsto h(x)-C$ is convex and that $\Sigma$ is complete. Then the following assertions are equivalent.

(i) (5.1) has a local error bound at $\bar{x}$.

(ii) There exist $\tau, \delta \in(0,+\infty)$ such that

$$
N(\Sigma, x) \cap B_{X^{*}} \subset \tau h^{\prime}(x)^{*}\left(N(C, h(x)) \cap B_{Y^{*}}\right) \quad \forall x \in \Sigma \cap B(\bar{x}, \delta) .
$$

(iii) There exist $\tau, \delta \in(0,+\infty)$ such that (5.3) and (5.4) hold for all $x \in \Sigma \cap B(\bar{x}, \delta)$.

(iv) There exist $\tau, \delta \in(0,+\infty)$ such that (5.11) holds for all $x \in \Sigma_{0} \cap B(\bar{x}, \delta)$, where $\Sigma_{0}:=\{x \in \Sigma$ : $h(x) \notin$ $\operatorname{int}(C)\}$.

(v) There exist $\tau, \delta \in(0,+\infty)$ such that (5.3) and (5.4) hold for all $x \in \Sigma_{0} \cap B(\bar{x}, \delta)$. 
(vi) There exist $\tau, \delta \in(0,+\infty)$ such that (5.3) holds and

$$
d\left(0, E\left(h^{\prime}(x)^{*}\left(\Sigma_{Y^{*}} \cap N(C, h(x))\right)\right) \geq \frac{1}{\tau}\right.
$$

for all $x \in \Sigma_{0} \cap B(\bar{x}, \delta)$.

(vii) There exist $\tau, \delta \in(0,+\infty)$ such that

$$
d(h, T(\Sigma, x)) \leq \tau d\left(h^{\prime}(x)(h), T(C, h(x))\right)
$$

for all $(h, x) \in X \times\left(\Sigma_{0} \cap B(\bar{x}, \delta)\right)$.

Remark. Using the fact that $C+C^{\infty}=C$, it is easy to verify that Assumption (A4) of Theorem 5.1 implies that the multifunction $x \mapsto h(x)-C$ is convex. Hence, (i) $\Leftrightarrow$ (iii) of Theorem 5.4 extends Theorem 5.1 of Burke and Deng [3].

Finally we extend Theorem 5.2. For this, we need the following lemma.

Lemma 5.5. Let $A$ be a finite subset of a normed space $Y$ and let $x \in E(\operatorname{co}(A))$. Then, there exists a subset $A_{0}$ of $A$ such that $x \in \operatorname{co}\left(A_{0}\right)$ and $0 \notin \operatorname{co}\left(A_{0}\right)$.

Proof. Let $\mathscr{A}$ denote the family of all faces of $\operatorname{co}(A)$ containing $x$. Since $\operatorname{co}(A)$ is a polyhedron of finite dimension, $\mathscr{A}$ consists of finitely many polyhedra. It is easy to verify that $\mathscr{A}$ has the least set, say $P_{0}$. Thus, there exists a subset $A_{0}$ of $A$ such that $P_{0}=\operatorname{co}\left(A_{0}\right)$. If $A_{0}=\{x\}$, the assertion trivially holds. Now suppose that $A_{0} \neq\{x\}$. We claim that $x \in \operatorname{ri}\left(P_{0}\right)$, where $\operatorname{ri}\left(P_{0}\right)$ denotes the relative interior of $P_{0}$. Granting this, by $x \in E(\operatorname{co}(A))$, it is easy to verify that 0 is not in $\operatorname{co}\left(A_{0}\right)$. It remains to show that $x \in \operatorname{ri}\left(P_{0}\right)$. Indeed, if this is not the case, then there exists a face $Q$ of $P_{0}$ such that $x \in Q$ and $Q \cap \operatorname{ri}\left(P_{0}\right)=\emptyset$. It follows that $Q \in \mathscr{A}$, contradicting the fact that $P_{0}$ is the least set of $\mathscr{A}$. This completes the proof.

In the case when $X=\mathbb{R}^{n}, Y=\mathbb{R}^{m}, C=-\mathbb{R}_{-}^{m}$ and $h(x)=\left(f_{1}(x), \ldots, f_{m}(x)\right)$ for all $x \in X$ where each $f_{i}$ is a smooth and convex function on $X$, Proposition 5.6 reduces to Theorem 5.2.

Proposition 5.6. Let $\Sigma, \bar{x}$ and $h$ be as in Theorem 5.4. Suppose that there exists an open neighborhood $V$ of $h(\bar{x})$ such that

$$
C \cap V=\left\{y \in Y:\left\langle y_{i}^{*}, y\right\rangle \leq r_{i}, i \in I\right\} \cap V,
$$

where $y_{i}^{*} \in Y^{*}, r_{i} \in \mathbb{R}$ and $I=\{1, \ldots, m\}$. Further suppose that there exists $\delta_{0}>0$ such that $h\left(B\left(\bar{x}, \delta_{0}\right)\right) \subset V$. Then (5.1) has a local error bound at $\bar{x}$ if and only if there exists a neighborhood $U$ of $\bar{x}$ such that (5.3) holds for all $x \in \Sigma \cap U$.

Proof. Since the necessity part is immediate from (i) $\Rightarrow$ (iii) of Theorem 5.4, we need only show the sufficiency part. Suppose that there exists a neighborhood $U$ of $\bar{x}$ such that (5.3) holds for all $x \in \Sigma \cap U$. Let $P:=\{y \in$ $\left.Y:\left\langle y_{i}^{*}, y\right\rangle \leq r_{i}, i \in I\right\}, I(y):=\left\{i \in I:\left\langle y_{i}^{*}, y\right\rangle=r_{i}\right\}$ for each $y \in P$. Then, by (5.14),

$$
N(C, y)=N(P, y)=\mathbb{R}_{+} \operatorname{co}\left\{y_{i}^{*}: i \in I(y)\right\} \quad \forall y \in V .
$$

Take $\delta_{1} \in\left(0, \delta_{0}\right)$ such that $B\left(\bar{x}, \delta_{1}\right) \subset U$. Then, (5.3) holds for all $x \in \Sigma \cap B\left(\bar{x}, \delta_{1}\right)$ and

$$
N(C, h(x))=\mathbb{R}_{+} \operatorname{co}\left\{y_{i}^{*}: i \in I(h(x))\right\} \quad \forall x \in \Sigma \cap B\left(\bar{x}, \delta_{1}\right)
$$

By (vi) $\Rightarrow$ (i) of Theorem 5.4, it suffices to show that there exist $\delta, \tau \in(0,+\infty)$ such that (5.12) holds for all $x \in \Sigma \cap B(\bar{x}, \delta)$. To do this, we show that there exists $\delta \in\left(0, \delta_{1}\right)$ and $\eta>0$ such that

$$
\eta<d\left(0, E\left(\operatorname{co}\left\{h^{\prime}(x)^{*}\left(y_{i}^{*}\right): i \in I(h(x))\right\}\right)\right) \quad \forall x \in \Sigma \cap B(\bar{x}, \delta)
$$


Indeed, if this is not the case, then there exist a sequence $\left\{x_{k}\right\}$ in $\Sigma \cap B\left(\bar{x}, \delta_{1}\right)$ convergent to $\bar{x}$ and a sequence $\left\{u_{k}^{*}\right\}$ in $X^{*}$ convergent to 0 such that

$$
u_{k}^{*} \in E\left(\operatorname{co}\left\{h^{\prime}\left(x_{k}\right)^{*}\left(y_{i}^{*}\right): i \in I\left(h\left(x_{k}\right)\right)\right\}\right) \text { for all } k .
$$

Since $I$ is finite and each $I\left(h\left(x_{k}\right)\right)$ is a subset of $I$, by Lemma 5.5 we can assume that there exists a subset $I_{0}$ of $I$ such that $I_{0} \subset I\left(h\left(x_{k}\right)\right), u_{k}^{*} \in \operatorname{co}\left\{h^{\prime}\left(x_{k}\right)^{*}\left(y_{i}^{*}\right): i \in I_{0}\right\}$ and

$$
0 \notin \operatorname{co}\left\{h^{\prime}\left(x_{k}\right)^{*}\left(y_{i}^{*}\right): i \in I_{0}\right\}
$$

for each $k$ (take a subsequence of $\left\{x_{k}\right\}$ if necessary). Thus, for each $k$ there exists $v_{k}^{*} \in \operatorname{co}\left\{y_{i}^{*}: i \in I_{0}\right\}$ such that $u_{k}^{*} \in h^{\prime}\left(x_{k}\right)^{*}\left(v_{k}^{*}\right)$. Noting (by (5.15)) that $v_{k}^{*} \in N\left(C, h\left(x_{k}\right)\right)$ and by Lemma 5.3 (applied to $F$ defined there, and to $x_{k}$ in place of $\left.\bar{x}\right)$, we conclude that $u_{k}^{*} \in D^{*} F\left(x_{k}, 0\right)\left(v_{k}^{*}\right)$, that is,

$$
\left(u_{k}^{*},-v_{k}^{*}\right) \in N\left(\operatorname{gph}(F),\left(x_{k}, 0\right)\right) .
$$

Since $\operatorname{co}\left\{y_{i}^{*}: i \in I_{0}\right\}$ is compact, $\left\{v_{k}^{*}\right\}$ has a subsequence $\left\{v_{k_{j}}^{*}\right\}$ convergent to some $v^{*} \in \operatorname{co}\left\{y_{i}^{*}: i \in I_{0}\right\}$. Hence, $\left(u_{k_{j}}^{*},-v_{k_{j}}^{*}\right) \rightarrow\left(0,-v^{*}\right)$. It follows from (5.18) and the convexity of $F$ that $\left(0,-v^{*}\right) \in N(\operatorname{gph}(F),(\bar{x}, 0))$. Noting that

$$
\left\langle\left(0,-v^{*}\right),(\bar{x}, 0)\right\rangle=\left\langle\left(0,-v^{*}\right),\left(x_{k}, 0\right)\right\rangle=0,
$$

it follows from the convexity of $F$ that $\left(0,-v^{*}\right) \in N\left(\operatorname{gph}(F),\left(x_{k}, 0\right)\right)$. This and Lemma 5.3 imply that $h^{\prime}\left(x_{k}\right)^{*}\left(v^{*}\right)=0$, contradicting (5.17). Hence there exist $\delta \in\left(0, \delta_{1}\right)$ and $\eta \in(0,+\infty)$ such that (5.16) holds. Let $M:=\max \left\{\left\|y_{i}^{*}\right\|: i \in I\right\}$. Then, from (5.15) and the definition of the end set $E(\cdot)$, it is easy to verify that for any $x \in \Sigma \cap B(\bar{x}, \delta)$,

$$
\begin{aligned}
d\left(0, E\left(\operatorname{co}\left\{h^{\prime}(x)^{*}\left(y_{i}^{*}\right): i \in I(h(x))\right\}\right)\right) & =d\left(0, E\left(h^{\prime}(x)^{*}\left(\operatorname{co}\left\{y_{i}^{*}: i \in I(h(x))\right\}\right)\right)\right) \\
& \leq d\left(0, E\left(h^{\prime}(x)^{*}\left(M B_{Y^{*}} \cap N(C, h(x))\right)\right)\right) \\
& =M d\left(0, E\left(h^{\prime}(x)^{*}\left(\Sigma_{Y^{*}} \cap N(C, h(x))\right)\right)\right) .
\end{aligned}
$$

This and (5.16) imply that (5.12) holds with $\tau=\frac{M}{\eta}$ for all $x \in \Sigma \cap B(\bar{x}, \delta)$. This completes the proof.

\section{REFERENCES}

[1] J.F. Bonnans and A. Shapiro, Perturbation Analysis of Optimization Problems. Springer, New York (2000).

[2] J.V. Burke and S. Deng, Weak sharp minima revisited, Part I: Basic theory. Control Cybern. 31 (2002) 399-469.

[3] J.V. Burke and S. Deng, Weak sharp minima revisited, Part III: Error bounds for differentiable convex inclusions. Math. Program. 116 (2009) 37-56.

[4] P.L. Combettes, Strong convergence of block-iterative outer approximation methods for convex optimzation. SIAM J. Control Optim. 38 (2000) 538-565.

[5] A.L. Dontchev and R.T. Rockafellar, Regularity and conditioning of solution mappings in variational analysis. Set-Valued Anal. 12 (2004) 79-109.

[6] A.L. Dontchev, A.S. Lewis and R.T. Rockafellar, The radius of metric regularity. Trans. Amer. Math. Soc. 355 (2003) $493-517$.

[7] R. Henrion and A. Jourani, Subdifferential conditions for calmness of convex constraints. SIAM J. Optim. 13 (2002) $520-534$.

[8] R. Henrion and J. Outrata, Calmness of constraint systems with applications. Math. Program. 104 (2005) 437-464.

[9] R. Henrion, A. Jourani and J. Outrata, On the calmness of a class of multifunctions. SIAM J. Optim. 13 (2002) 603-618.

[10] H. Hu, Characterizations of the strong basic constraint qualification. Math. Oper. Res. 30 (2005) 956-965.

[11] H. Hu, Characterizations of local and global error bounds for convex inequalities in Banach spaces. SIAM J. Optim. 18 (2007) 309-321.

[12] A.D. Ioffe, Metric regularity and subdifferential calculus. Russian Math. Surveys 55 (2000) 501-558.

[13] D. Klatte and B. Kummer, Nonsmooth Equations in Optimization, Regularity, Calculus, Methods and Applications; Nonconvex Optimization and its Application 60. Kluwer Academic Publishers, Dordrecht (2002).

[14] A. Lewis and J.S. Pang, Error bounds for convex inequality systems, in Generalized Convexity, Generalized Monotonicity: Recent Results, Proceedings of the Fifth Symposium on Generalized Convexity, Luminy, June 1996, J.-P. Crouzeix, J.-E. Martinez-Legaz and M. Volle Eds., Kluwer Academic Publishers, Dordrecht (1997) 75-100. 
[15] W. Li, Abadie's constraint qualification, metric regularity, and error bounds for differentiable convex inequalities. SIAM J. Optim. 7 (1997) 966-978.

[16] W. Li and I. Singer, Global error bounds for convex multifunctions and applications. Math. Oper. Res. 23 (1998) $443-462$.

[17] B.S. Mordukhovich, Complete characterization of openness, metric regularity, and Lipschitzian properties of multifunctions. Trans. Amer. Math. Soc. 340 (1993) 1-35.

[18] K.F. Ng and X.Y. Zheng, Error bound for lower semicontinuous functions in normed spaces. SIAM J. Optim. 12 (2001) 1-17.

[19] S.M. Robinson, Regularity and stability for convex multivalued fucntions. Math. Oper. Res. 1 (1976) 130-143.

[20] C. Zalinescu, Weak sharp minima, well-behaving functions and global error bounds for convex inequalities in Banach spaces, in Proc. 12th Baical Internat. Conf. on Optimization Methods and their applications, Irkutsk, Russia (2001) $272-284$.

[21] C. Zalinescu, Convex Analysis in General Vector Spaces. World Scientific, Singapore (2002).

[22] C. Zalinescu, A nonlinear extension of Hoffman's error bounds for linear inequalities. Math. Oper. Res. 28 (2003) 524-532.

[23] X.Y. Zheng and K.F. Ng, Metric regularity and constraint qualifications for convex inequalities on Banach spaces. SIAM J. Optim. 14 (2003) 757-772.

[24] X.Y. Zheng and K.F. Ng, Metric subregularity and constraint qualifications for convex generalized equations in Banach spaces. SIAM. J. Optim. 18 (2007) 437-460.

[25] X.Y. Zheng and K.F. Ng, Linear regularity for a collection of subsmooth sets in Banach spaces. SIAM J. Optim. 19 (2008) $62-76$. 